\title{
Managing variability: a cognitive ethnography of the work of airline dispatchers
}

\author{
Pamela Munro, M.A. \\ Feiji Consulting \\ Seattle, WA \\ Richard Mogford, Ph.D. \\ NASA Ames Research Center \\ Moffett Field, CA
}

\begin{abstract}
Airline dispatchers' workflow is often described in broad terms like 'flight planning' and 'flight following'. Such high-level descriptions fail to recognize the number and complexity of tasks involved in these activities. An ethnographic study was conducted at three US airlines to understand the cognitive workload involved in flight planning. Fuel planning was identified as one of five key flight planning tasks. Fuel planning was conducted concurrently with other planning and monitoring tasks which often led to interruptions. Planning fuel was dynamic, with recalculations required whenever other factors varied (e.g., payload, route, alternates). This rework increased workload and opportunities for error while reducing efficiency. Four main factors contributed variability to fuel planning: contingency planning, load planning, pilots, and station operations. Strategies for managing variability included pattern identification, use of buffers, rounding up, and leveraging software tools. Software design often added to workload by forcing dispatchers to attend to low level tasks.
\end{abstract}

\section{INTRODUCTION}

Airline dispatchers play a critical role in ensuring the safety of commercial aviation. As defined in Title 14 of the Code of Federal Regulations (14 CFR) domestic and flag operations, the dispatcher shares responsibility for safety and operational control for any flight under his/her guidance with the pilot in command (PIC) (c.f., FARs 121.533,121.535). Operational control is defined as "authority over initiating, conducting, or terminating a flight" (14 CFR Part 1.1). In daily airline operations, this includes responsibility for tasks such as the preflight planning, dispatch release, flight monitoring and, if necessary, delay of a flight. Dispatchers also interact with air traffic control (ATC), filing flight plans and coordinating routing. Indeed the relationship between dispatch, pilots, and ATC has often been described as a triad in which all three elements must work together to ensure safe and efficient operations.

Despite playing such an important role in the safety and efficiency of commercial aviation, the focus in much of the research literature has been on the tangible output of dispatchers' work, i.e., the flight plan, and the way that it becomes part of the workflow of others (such as pilots and air traffic controllers) with little focus on the workload and workflow that went into producing it.

\section{Duties}

Dispatchers' workflow is frequently described in broad terms like 'flight planning' and 'flight following'. Flight planning is generally defined as determining the route that will avoid weather and other hazards while ensuring it has enough fuel to do so. Similarly, flight following is broadly thought of as monitoring a flight from take off until landing and giving pilots updates on weather. Such high-level descriptions of dispatchers' work fail to recognize the number and complexity of tasks involved in each of these activities.

At a task level, for example, flight planning involves seeking out information relevant to a flight (including but not limited to: weather, aircraft capabilities, runway configuration, airspace, station operations, and flight crew qualifications), assessing the potential impact of these pieces of information (individually and collectively) on the successful conduct of a flight, then building a plan that mitigates any such impact while also providing the pilot with sufficient resources to deal with any unforeseen events.

At a cognitive level, it involves skills such as perception, attention, memory, situation awareness, pattern-recognition, decision-making, and multi-tasking, each of which contributes to dispatchers' human performance capabilities but also introduces limitations and biases that can lead to errors. All of this is performed in an environment that includes stressors such as time pressure, productivity goals, and the risk of professional liability for decisions made. Further, it often takes place on the 'back side of the clock' which brings risks associated with fatigue (FAA, 2010).

\section{Cognitive ethnography}

The aim of cognitive ethnography is to reveal how cognitive activities are accomplished in the real-world. A central tenet of this approach is that cognition cannot be studied without awareness of its cultural and environmental settings, social interactions and communication, use of artifacts, and even the ways in which participants use their own bodies to construct and share meaning. It rests on a theoretical foundation of distributed cognition, situated action, and cultural psychology (cf Hutchins,1996; Suchman,1987; Star and Strauss, 1999). 
The goal of a cognitive ethnographic study is to describe how knowledge is actively constructed and used by a particular community of practice (Williams, 2006). To accomplish this a researcher must spend significant time immersed in that community building knowledge about their cultural values and practices and applying this to the analysis of their cognitive activity (Lewis, 1985). Such analysis focuses on uncovering what is meaningful to practitioners as they perform cognitive activities and how that meaning is actively constructed in the moment of action.

\section{Present study}

A series of field studies were conducted to capture the work practices of airline dispatchers as well as the situational, social and environmental factors and artifacts that make up their work environment. Of particular interest was building a more complete understanding the cognitive demands involved in flight planning and identifying the strategies dispatchers developed to manage this cognitive workload.

\section{METHOD}

\section{Procedure}

A series of ethnographic field observations were conducted at three US airlines over a period of five months. Researchers observed and interacted with line dispatchers as they planned, released, and monitored flights. Additional observations were conducted with chief dispatchers and ATC coordinators. Data were captured via field notes, photographs, and video recordings created during observation sessions. In addition variety of artifacts including operational memos, flight assignment sheets, ATC updates, and dispatcher-created notes were also collected to provide further insights into how dispatchers organize and manage their work.

Semi-structured interviews were conducted to collect data about each dispatcher's current position, prior experience and training, the tools and processes of their airline, and the strategies and techniques they employed for managing information and workload. When possible audio or video recordings were made to ensure dispatcher comments were accurately captured.

\section{Participants}

Dispatchers. Scheduled participants included a total of 15 line dispatchers, chief dispatchers, and air traffic coordinators. Participants ranged in age from early $20 \mathrm{~s}$ to late $60 \mathrm{~s}$ and included 9 males and 6 females. Experience working as a dispatcher ranged from 3 years to 35 years. Participants were observed working domestic and international desks during day and swing (afternoon into evening) shifts.

Airlines. Observations were conducted at three major US airlines. Two airlines were flag carriers operating a variety of aircraft types across extensive international networks. The third operated a single aircraft type on short and long-haul routes in throughout North America and between the mainland and Hawaii.

\section{Data analysis}

Following each observation session, field notes were expanded into fully fleshed-out accounts or 'thick descriptions' (Geertz, 1973) of the events, actions, tasks, environments, interactions, and participant comments that were observed. Any audio or video recordings were reviewed and transcribed, and photographs, operational memos, dispatcher-created notes and other collected artifacts were also incorporated to provide as complete a representation of each session's events as possible (Emerson, Fretz, and Shaw, 2011)

Once all observation sessions were completed, the full corpus of data was analyzed using a grounded theory approach. Grounded theory is an inductive approach through which themes emerge from the data collected rather than from pre-determined hypotheses (Glaser and Strauss, 2000). In the first phase of analysis, use of an open-coding process was used to identify patterns within the data. These patterns were then developed into a formal coding scheme. The second phase of analysis employed a process of focused coding in which this coding scheme was used to re-analyze the full corpus of data. From this process refinements were made to the coding scheme itself (e.g., combining overlapping categories into a single category, breaking out an overly-broad category into several more focused categories) as well as more detailed definition of the main themes revealed in the data.

As themes were identified at each level of analysis, a search for and review of relevant literature was conducted to identify where coded themes aligned with previous studies, where they did not, and where there were gaps in the existing research.

\section{RESULTS}

\section{Flight planning}

The planning of a flight must necessarily be accomplished prior to the actual conduct of that flight. As a result, the flight's plan is based on a number of estimations or predictions about the conditions in which the flight will take place, e.g., forecast weather, estimated passenger loads, predicted delays, expected air traffic programs. Any one of these conditions can change at any time before the flight departs. Many can also change once the flight is enroute. Each change requires the dispatcher to review the new information, assess its impact to the existing plan, and identify the best strategy for mitigating that impact. Thus a dispatcher may revisit the same plan repeatedly over the lifecycle of a flight. Flight planning is therefore best characterized as ongoing, dynamic process rather than as a linear task with discrete steps that lead to a fixed output.

Similarly, a dispatcher's workflow cannot always be neatly divided into discrete phases of flight planning or flight following. Because each dispatcher in this study was responsible for multiple flights over the course of a shift, planning for some flights was conducted concurrently with following other flights. One of the main cognitive challenges for dispatchers was this ongoing multi-tasking, i.e. the need to switch back and forth between planning and following 
multiple flights while remembering the constraints and goals for each. It placed significant demands on resources like attention and memory and challenged situation awareness.

Flight plan versus dispatch release. The term flight plan is often used to refer to what is properly called the dispatch release. The flight plan (also called the flight strip), is only one part of the dispatch release. It contains the proposed route of flight and is submitted to ATC for approval before departure. The dispatch release, on the other hand, includes all aspects of the operational plan (route, fuel, payload, performance or mechanical restrictions, etc.) and the information used to create it, e.g., weather forecasts, fuel calculations, deferred mechanical items, passenger and cargo loads, Notices to Airmen (NOTAMs), in addition to the proposed route.

Once he/she has completed flight planning the dispatcher 'releases' the plan, i.e., makes it available to other work groups throughout the airline (e.g., pilots, load planners, ramp controllers, etc.), who use it to support their own work processes. Many operations cannot begin until the release is available. For example, ramp controllers cannot generate the fuel slips that must be sent to fuel trucks in order to begin fueling the aircraft until the total fuel quantity needed is received via the release. As they begin working with the release these work groups may discover some of the predictions on which it was based were not accurate or that situational factors have changed. They communicate this information to the dispatcher who must amend and update the release.

Schedule release time. Each dispatch release has an assigned time by which it must be completed, known as the 'schedule release time'. Airlines in this study had schedule release times of between 60-90 minutes before domestic departures and 120 minutes before international departures. A dispatcher can, however, choose to release a flight earlier than the schedule release time. Indeed this was often done when a dispatchers had multiple releases due at roughly the same time. Completing some releases early helped ensure there was sufficient time available to plan each flight. Other times a dispatcher was aware that a station would need additional time to prepare a particular flight and finishing the planning early gave them the extra time. Flight plans for international flights could be completed up to several hours in advance, in part because they needed to be submitted to all the air traffic service providers through whose airspace the flight would pass for review and approval.

Dispatchers were sensitive to meeting their airline's release times not only because it was a metric on which their own performance was evaluated, but also because they were aware the impact a late release had on other work groups. (Indeed it was observed that some stations would telephone the dispatcher when a release went as few as five minutes beyond its scheduled time). Flight plans were generally sent to ATC 45-60 minutes before departure for domestic flights and 60-90 minutes for international flights.

Key flight planning tasks. There are a number of activities involved in planning a flight. This study identified five key planning tasks: checking weather, choosing a route, selecting alternates, reviewing NOTAMs, and planning fuel. In practice these tasks are tightly interwoven and often performed concurrently. However examining them individually allows us to identify what is unique to each and what they may have in common. The rest of this paper will focus specifically on the activities involved in planning fuel.

\section{Fuel planning}

Fuel planning involves calculating not only the amount of fuel necessary to fly from the departure to destination airports along the selected route, but also the amounts needed for each phase of operation, such as taxi out, taxi in, holding, diversion to an alternate, etc. The plan must further demonstrate that upon landing the flight will meet the minimum reserve fuel requirements (45 minutes flying time). The dispatcher must also verify that the planned fuel will not put the aircraft over any structural and performance limits (e.g., max takeoff weight, max landing weight).

Fuel was observed to be the factor dispatchers revisited the most throughout the flight planning process. Any time a factor on which the original plan had been built changed, the fuel plan had to be re-assessed. Four main contributors of variability were identified: contingency planning, load planning, pilots, and station operations. These will be examined individually.

Contingency planning. Dispatchers consistently identified fuel as the one factor impacting flight safety over which they had direct input. One of dispatchers' greatest concerns was the risk of missing some small detail in the reams of data they must review while planning a flight, e.g., an airspace restriction, a fuel penalty in an MEL, or a detail in a weather forecast that could negatively impact the flight. Even if no details were missed, dispatchers knew from experience that events out of their control routinely occurred during revenue flights (e.g., ATC clearances, weather, mechanical issues).

Ensuring the flight crew had sufficient contingency fuel to successfully manage any such events was a top priority for dispatchers. To accomplish this they quite frequently rounded up individual fuel quantities to add an additional margin of safety. For example, one dispatcher who had calculated that 270 pounds of fuel would be needed for taxi out rounded the quantity up to an even 300 pounds. This strategy was also applied to each of the other planned fuel quantities for that flight (e.g., taxi, enroute, holding, diversion, etc.) After summing these individual fuel quantities to get a total fuel quantity, dispatchers would frequently round up the total fuel number to add an additional margin for contingencies.

Load planning. Dispatchers often began flight planning using an initial estimate of the aircraft's zero fuel weight (ZFW) provided by the airline's load planners. The ZFW is the weight of the airplane plus everything to be loaded on it, with the exception of fuel (e.g., crew, catering, passengers, cargo, etc.). When added together the ZFW and the total fuel cannot exceed the most limiting weight determined for a flight (whether structural or performance based). The initial ZFW estimate is based on historical loads, seasonal factors, and other operational data. While a common operational target was to have load planning provide dispatch with the actual ZFW twenty minutes before departure, it was not uncommon for 
final load numbers to be provided only after the aircraft door had closed.

The ZFW is recorded in the airline's flight tracking tool along with other data about a flight. At all three airlines this tracking tool consisted of a spreadsheet in which each row represented a single flight and each column represented a different piece of data about that flight. (See Figure 1). The average number of columns per flight was twenty-seven. As one dispatcher commented, "Everything we do is numbers." The only text-based fields were those for airport identifiers and a 'Notes' field which allowed a limited amount of free text.

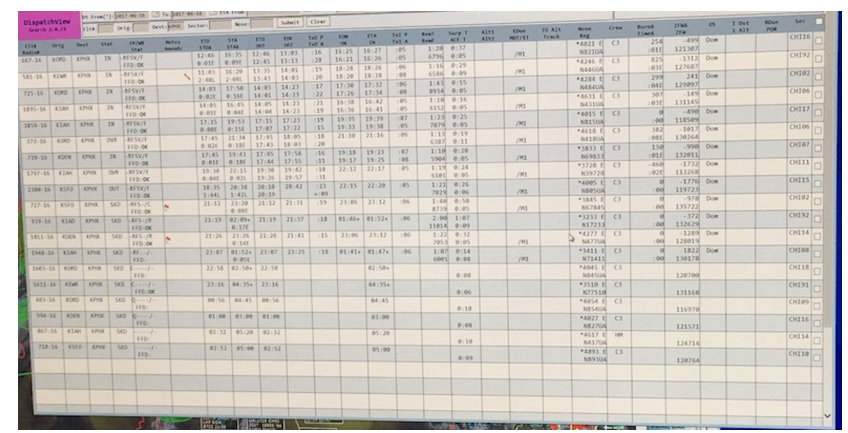

Figure 2. Example of a flight data tracking tool.

Although dispatchers expected that the ZFW would fluctuate as payload figures became more refined leading up to departure, the only visual indication that a ZFW had changed was a change in the numbers in the ZFW field. It was not uncommon for this to occur while the dispatcher's

attention was focused on other tasks. The pressure to meet schedule release times makes it impossible for a dispatcher to focus only on the ZFW of one flight at a time. To track changes dispatchers could attempt to memorize the current ZFW for each flight, but the well-documented vulnerabilities of short-term memory make this a risky strategy. Writing each ZFW on a piece of paper would offload some of the memory burden while bringing a host of other vulnerabilities.

Ultimately the strategy dispatchers found most effective for monitoring changes to the ZFW was leveraging affordances of the flight tracking tool itself. They would copy and paste, or manually type, the most recent ZFW value for a flight into the flight's Notes field. This eliminated the need to remember the value and made it possible to compare the number in the Notes field with the number in the ZFW field at any point to determine if there had been a change. This reduced cognitive workload by offloading the memory burden onto the software tool, and turned detecting a change from a calculation task to a visual task. (However it still required the dispatcher to remember to periodically check the tool).

Once a change was detected, the dispatcher would need to re-calculate fuel quantities. Recalculating fuel interrupted other planning tasks, increased the time needed to complete each release, introduced opportunities for error, and added to overall dispatcher workload. To eliminate the need to recalculate with every small fluctuation, dispatchers added buffers to the initial ZFW. At Airline A there was a wellknown and accepted practice for dispatchers to add 1,000 pounds to the initial ZFW received from load planning. In this way if load planning needed, for example, 800 additional pounds for cargo or passengers, this could be accommodated without requiring the dispatcher to recalculate fuel.

Interestingly, load planners at the airline reported they had a practice of adding their own 1,000-pound buffer to the ZFW before sending it to dispatch as a means of holding space for additional payload. Thus fuel planning began with a total of 2,000-pounds of buffer built into the ZFW. While this slightly reduced the available weight capacity for fuel, it absorbed the impact of many fluctuations in ZFW, eliminating the need for the dispatcher to recalculate each time. When a flight showed very close to its maximum weight limit, however, it required remembering how much of the added buffer had actually been used.

While the regulations hold dispatchers responsible (along with the PIC) for planning enough fuel to safely conduct the flight (with adequate margins for unforeseen events), airlines sent clear messages to dispatchers that they were also expected to support company business goals by keeping fuel costs down and by accommodating more paying passengers or cargo.

As a result it was frequently observed that determining how much of an aircraft's available weight capacity would be allocated to fuel versus payload was a process of ongoing negotiation between dispatchers and load planners. Dispatchers were often encouraged to find ways to accommodate more revenue-generating payload (e.g., by adjusting fuel allocations, the cost index, estimates for holding, etc.). During one session a dispatcher, frustrated after receiving a series of phone calls from load planning requesting more payload demanded, "Just tell me what you need!"

Pilots. Load planning was not the only source of variability to fuel planning. Once the dispatcher releases a flight, the captain, as PIC, must review the plan and decide whether to accept it as-is or request changes. One of the most common changes captains requested was additional fuel. Requests typically came during telephone briefings with the dispatcher or via ACARS messages once pilots were onboard.

To minimize the need to recalculate dispatchers sought ways to account for pilot requests earlier in the planning process. A common method was to track the fuel requests from captains with whom they regularly worked. It turned out most were quite consistent in their requests, which allowed dispatchers to include each captain's preference from the start of their planning. As one dispatcher commented, "If they ask for more fuel I'll always give it to them. Ultimately they're the ones in the hot seat out there."

Station operations. Agents at airports regularly attempted to accommodate additional passengers on flights with available seats right up until departure. This increased the ZFW which required dispatchers to recalculate fuel with only minutes until departure. Rushing fuel calculations increases opportunities for error, while taking extra time risks a schedule delay. Dispatchers sought ways to track these types of changes in order to account for them earlier in the planning process. One dispatcher explained how, after having to make last-minute recalculations on one of his regular flights several times in a row, he checked with the station and learned that a commuter airline had a flight that arrived shortly before his mainline flight's departure. To provide good customer service, 
the gate agents accommodated connecting passengers until door closure. The dispatcher tracked the extra weight and calculated an average that he would add to the flight's ZFW at the start of his planning. This allowed the agents to continue supporting company goals while reducing the additional workload and risks caused by last-minute fuel recalculations.

\section{DISCUSSION}

Dispatchers play a critical role in the safety of commercial aviation. They are responsible for planning, releasing, and monitoring of flights. They form part of the larger airline operations team that also includes pilots, station operations, and load planning. Yet studies of the daily cognitive demands of dispatchers' work are often missing from the research literature. This study sought to capture the workflow and cognitive demands involved in flight planning by means of an ethnographic field study.

Fuel planning was one of five key tasks identified as part of the flight planning process. One of the main challenges when planning fuel is that the data on which the initial fuel plan is built will continue to change right up until departure. Four main sources of this input variability were identified: pilots, load planning, station operations, and contingency planning.

Each of these sources contributes to variability by adding information, changing existing information or by shifting operational goals. For example, pilots may request additional fuel based on their experience; a station may want to change the number of passengers onboard; load planning may add additional payload. Each change requires the dispatcher to reassess and re-calculate fuel loads to ensure the flight can be conducted safely and efficiently. Each recalculation adds to the time necessary to complete a dispatch release, which can jeopardize meeting the dispatch release time and can reduce a dispatcher's overall productivity. Re-calculating also interrupts workflow and pulls attentional resources from other planning and monitoring tasks, both of which can increase cognitive workload.

To reduce disruption to their workflow and additional cognitive demands, dispatchers developed a number of strategies. The common theme across all strategies was identifying and accounting for variability as early as possible in the planning process in order to manage them proactively rather than reactively. A fundamental strategy was detecting reliable patterns in the way fuel planning variables changed. Identifying such patterns allowed dispatchers to predict changes in advance and build some form of resilience into the fuel plan that could absorb changes without requiring active recalculation by the dispatcher.

Software tools are not always designed with an understanding of the dynamic nature of flight planning information and dispatchers' need to respond in some way to each change. As a result software tools often add workload, for example by requiring dispatchers to focus attention on tasks like monitoring changes in numeric fields. Such lowlevel activities divert attention away from higher-level activities like decision-making and maintaining situation awareness. They also increase the time it takes to complete a flight plan, decreasing efficiency. To truly mitigate workload, designers must understand that dispatch work is full of stops and starts, interruptions, and changes, and that planning software must be designed to support tasks like place-keeping, filtering, and tracking changes to help reduce errors and allow dispatchers to put more attention on higher-level cognitive tasks.

As airlines continue to add more flights, finding ways to reduce dispatchers' cognitive workload becomes more critical. The greater the number of flights a dispatcher must plan, the less time s/he can spend planning each one. As a dispatcher planning domestic long haul flights explained, "The more flights you give me to work, the more conservative I become, because I have less time to work each one and consider all the risks." The unintended consequence of this was explained by another dispatcher: "Fuel is the one thing we have control over that has a direct impact on safety. If I can't consider everything, I can at least put on more fuel." Thus when faced with more flights and less time to plan, dispatchers will increase the fuel quantities used in buffers and rounding up. This suggests that reducing dispatchers' cognitive workload can directly impact an airline's bottom line.

\section{REFERENCES}

Emerson, R., Fretz, R., and Shaw, L. (2011). Writing ethnographic fieldnotes. Chicago, IL: University of Chicago Press.

Federal Aviation Administration. (2010) Advisory Circular AC 120-100 Basics of aviation fatigue. Washington, DC: Author.

Federal Aviation Regulations. (2017), Federal Aviation Administration, Washington D.C.: Author.

Geertz, C. (1973). The interpretation of cultures: Selected essays. New York: Basic Books.

Glaser, B.G. and Strauss, A.L. (2000). The discovery of grounded theory: Strategies for qualitative research. Abingdon-on-Thames, England: Routledge.

Hutchins, E. (1996). Cognition in the wild. Cambridge, MA: MIT Press.

Lewis, I. M. (1985). Social Anthropology in Perspective. Cambridge: Cambridge University Press.

Star, S. and Strauss, A. (1999). Layers of silence, arenas of voice: The ecology of visible and invisible work. Computer Supported Cooperative Work, 8, 9-30.

Suchman, L. (1987). Plans and situated actions. New York, NY: Cambridge University Press.

Williams, R. F. (2006). Using cognitive ethnography to study instruction. Proceedings of the 7th International Conference of the Learning sciences, Bloomington, Indiana: pp. $838-844$. 